\title{
Zum 100. Jahrgang des Forstwissenschaftlichen Centralblattes
}

Der 100. Jahrgang einer fachwissenschaftlichen Zeitschrift ist zwar gotrlob kein Einzelfall, aber für die unmittelbar Beteiligten - also die Herausgeber und den Verlag - doch kein alltägliches Ereignis, das man stillschweigend übergehen möchte. Hinzu kommt in diesem Falle, daß das Forstrissenschaftliche Centralblatt seit seiner Gründung im Jahre 1879 bis zum heutigen Tage im selben Verlag erscheint -- auch wenn dieser Verlag im Grundungsjahr der Zeitschrift noch als Verlag von Wiegandt, Hempel \& Parey firmierte - und daß die Zeitschrift von den Professoren der Forstwissenschaft an der Universität München der Hauptstadt des damaligen Königreiches Bayern - ausgerechnet in einem Verlag begründet wurde, der seinen Sitz in Berlin, der Hauptstadt des damaligen Königreiches PreuBen und seit wenigen Jahren auch des damaligen Deutschen Reiches, hatre. Daß die gemeinsame Arbeit für das Forstwissenschaftliche Centralblatt in all den Jahren von bayerischen Herausgebern und einem preußischen Verlag getragen wurde und diese vertrauensvolle Verbundenheit nunmehr hundert Jahre Bestand hat, sind Fakten, auf die alle, die für diese Zeitschrift in dieser Zeit in Verantwortung gestanden haben, wohl mit Recht ein wenig stolz sein dürfen und die beweisen, daß es mit den vermeintlich so unüberbrückbaren Gegensätzen zwischen Bayern und Preußen wohl doch nicht so weit her ist, jedenfalls dann nicht, wenn auf beiden Seiten - Herausgeber und Verlag - mit gutem Willen und steter Bereitschaft zum gegenseitigen Verständnis und zur Zusammenarbeit für eine gemeinsame Aufgabe gearbeitet wird.

Die wechselvolle Geschichte des Forstwissenschaftlichen Centralblattes ist Thema des in diesem 1. Heft des 100. Jahrganges zur Veröffentlichung gelangenden Festvortrages, den Richard Plochmann bei unserer kleinen akademischen Feier am 25. Februar dieses Jahres im Alten Senatssaal der Ludwig-Maximilians-Lniversität-München halten wird und in dem die Verdienste des Begründers und ersten Herausgebers dieser Zeitschrift Franz von Bauer (1879-1896) und seiner Nachfolger Herma.v von FúrST (1897-1914), MAX Endres (1915-1940), Vinzenz Schlipfer (1915-1942), Ludwic Fabricius (1915-1940), Kari. Vanselow (1942-1944), Hubert Freiherr von Pechimann (1948-1976) und all der anderen Persönlichkeiten, die sich um die Herausgabe des Forstwissenschaftlichen Centralblattes verdient gemacht haben, gewürdigt werden. Ihnen und allen jetzt und hier ungenannt bleibenden Mitherausgebern und Mitarbeitern der Zeitschrift und ihrem Andenken ist dieses Festhefr als ein außeres Zeichen des Dankes und der nachträglichen Anerkennung gewidmet. Ihrem Wirken fühlen wir uns an der Schwelle zum zweiten Jahrhundert des Forstwissenschaftlichen Centralblattes verpflichtet.

Die Ausführungen, mit denen sich der Gründungsherausgeber im ersten Heft der Zeitschrift an seine Leser wendet, zeigen mit einer uns heute vielleicht doch uberraschenden Deutlichkeit, wie gleichartig die Ziele dieser Zeitschrift damals wie heute waren. Schon damals war es das Anliegen der Gründer, das Forstwissenschaftliche Centralblatt zu einem gemeinsamen Sprachrohr aller Forstwissenschaftler im deutschen Sprachgebiet auszugestalten, das wechselseitig gediegene Informationen über die vielfältigen Aspekte des Waldes und der Forstwirtschaft vermittelt, zu einer Zeitschrift, die die aktuellen Probleme aufgreift und öffentlich zur wissenschaftlichen Diskussion stellt, die die Ergebnisse wissenschaftlicher Forschung der forstlichen Praxis in geeigneter Weise nahebringt und die durch 
die Veröffentlichung von Erfahrungen und Erkenntnissen der forstlichen Praxis die wissenschaftliche Arbeit befruchtet und aufzeigt, in welchen Bereichen diē Wissenschaft noch ungeklärte Probleme erforschen solle.

Bei aller Bedeutung von Tradition und Rückbesinnung auf Geleisteres dürfen sich jedoch die Gedanken derjenigen, die heute fur das Forstwissenschaftliche Centralblatt die Verantwortung tragen, nicht auf einen Rückblick beschränken. Die Probleme der Forstwirtschaft unterliegen einem ständigen Wandel. Es gibt kaum eine wissenschaftliche Disziplin, die so wie die Forstwissenschaft zukunftsorientiert arbeiten, denken, planen und forschen muß. Heute zu treffende Entscheidungen wirken sich in der Forstwirtschaft groBenteils erst in Jahrzehnten, ja, z. T. erst in hundert Jahren und mehr aus. Fehlentscheidungen, die heute gefält werden, haben infolgedessen besonders langfristige und häufig kaum noch zu revidierende negative Konsequenzen. Wir begrußen es daher dankbar, daß der zweite Festvortrag, den Gerhard Speidel am 25. Februar halten wird und der ebenfalls in diesem Festheft veröffentlicht wird, der gegenwärtigen Situation und den künftigen Problemen der forstwissenschaftlichen Forschung gewidmet ist. Nur die sorgfältige, kritische und vorurteilslose Analyse der gegenwärtigen Situation und der künftigen Probleme der Forstwirtschaft schaffr die Voraussetzungen für das Erkennen der richtigen Wege und der richrigen Entscheidungen. Technische und ökonomische Erwägungen allein - so wichtig und notwendig sie für die Forstwirtschaft nach wie vor sind und auch in Zukunft bleiben werden - reichen zur Bewältigung gegenwärtiger und künftiger Aufgaben nicht aus. Die Erhaltung des W'aldes - trotz aller nutzungsbedingter Eingriffe - als Erholungsbereich für den Menschen, als Lebensraum für die freilebende Tierwelt, als Klimafakior grolßflächiger Gebiete und als wichtigster Bestandteil einer natürlichen Landschaft und damit einer gesunden Umwelt stellt sich heute und für alle Zukunft der Forstwirtschaft und der Forstwissenschaft als eine gleichrangige, $z$. T. bereits vorrangige Aufgabe. Der Erforschung der biologischen und ökologischen Grundvoraussetzungen und -bedingungen des Waldes und der Forstwirtschaft in ihrer ganzen Vielfalt, in ihrer gegenseitigen Wechselwirkung und in ihrer Abhängigkeit hat daher unsere besondere Aufmerksamkeit zu gelten.

Die Herausgebergemeinschaft und die Verlagsbuchhandiung Paul Parey werden sich bemühen, der übernommenen und überkommenen Verantwortung für das Forstwissenschaftliche Centralblatt gereche zu werden. Sie empfinden es als ihre Verpflichtung, die Zeitschrift im Geiste ihrer Gründer und im Sinne ihrer Vorgänger so zu gestalten, daß die ihr in Gegenwart und Zukunft gestellten Aufgaben erfültt werden, damit der Forstwissenschaft und der Forstwirtschaft ein Publikationsorgan erhalten bleibt, das unserer Wissenschaft und damit zugleich dem Gemeinwohl dient. Wir danken allen denjenigen, die uns bei unserer Arbeit unterstüizen, insbesondere allen bei der Herausgabe der Zeitschrift mitwirkenden Persönlichkeiten, den Autoren und den Beziehern der Zeitschrift und hoffen auf weitere verrauensvolle und erfolgreiche Zusammenarbeic zum Nutzen unseres gemeinsamen Anliegens.

Berlin, Freiburg, Götringen, Hamburg und München am 1. Januar 1981

Ul.rich AMmer, Werner Kroth, Friedrich Georgi, Rudolf Georgi

Karl E. Rehifuess, Walter Schópfer,

Peter Schutt, Bernhard Ulricif 